\title{
Thermal Transport and Rectification Properties of Bamboo-Like SiC Polytypes Nanowires
}

\author{
Zan Wang \\ School of Mechanical and Electrical Engineering, Henan University of Technology, Zhengzhou 450007, China \\ Correspondence should be addressed to Zan Wang; truewz@163.com
}

Received 2 February 2017; Revised 3 May 2017; Accepted 10 May 2017; Published 11 June 2017

Academic Editor: David Cornu

Copyright (c) 2017 Zan Wang. This is an open access article distributed under the Creative Commons Attribution License, which permits unrestricted use, distribution, and reproduction in any medium, provided the original work is properly cited.

\begin{abstract}
Bamboo-like SiC nanowires (NWs) have specific geometric shapes, which have the potential to suppress thermal conductivity by phonon boundary scattering. In this work, phonon transport behaviors in the $3 \mathrm{C}-\mathrm{SiC}, 4 \mathrm{H}-\mathrm{SiC}$, and $6 \mathrm{H}-\mathrm{SiC}$ crystal lattices are studied by the Monte Carlo (MC) method, including impurity scattering, boundary scattering, and Umklapp scattering. Phonon relaxation times for Umklapp (U) scattering for the above three $\mathrm{SiC}$ polytypes are calculated from the respective phonon spectra, which have not been reported in the literature. Diffuse boundary scattering and thermal rectification with different aspect ratios are also studied at different temperatures. It is found that the thermal conductivities of the bamboo-like SiC polytypes can be lowered by two orders of magnitude compared with the bulk values by contributions from boundary scattering. Compared with bamboo-like $4 \mathrm{H}-\mathrm{SiC}$ and $6 \mathrm{H}-\mathrm{SiC} \mathrm{NWs}, 3 \mathrm{C}-\mathrm{SiC}$ has the largest $\mathrm{U}$ scattering relaxation rate and boundary scattering rate, which leads to its lowest thermal conductivities. The thermal conductivity in the positive direction is larger than that in the negative direction because of its lower boundary scattering relaxation rate.
\end{abstract}

\section{Introduction}

$\mathrm{SiC}$ is one of the most important third-generation semiconductors in the fields of electronic devices and hightemperature components. Particularly in harsh conditions, $\mathrm{SiC}$ devices are much better than $\mathrm{Si}$ and GaAs devices [1]. Though $\mathrm{SiC}$ has hundreds of stable polytypes, the most commonly used ones are $3 \mathrm{C}-\mathrm{SiC}, 4 \mathrm{H}-\mathrm{SiC}$, and $6 \mathrm{H}-\mathrm{SiC}$ [2]. In recent years, there have been many experimental and theoretical efforts invested in the development of $\mathrm{SiC}$ nanostructures [3-5]. Researchers [6-8] have proposed that thermal rectification effects are dependent on the size of the device and the asymmetric geometry. If phonons can be managed to transmit information as electrons and photons, thermal devices will also have more practical applications in phononic devices and the field of thermoelectric conversion.

To determine the boundary and interfacial effects of nanoscale structures, some groups have studied core-shell NWs [9], variable cross-section NWs $[10,11]$, and rough NWs $[12,13]$ in recent years. In thermal studies of $\mathrm{SiC}$, Ni et al. [14] and Chantrenne and Termentzidis calculated thermal conductivities and frequency density of states of (DOS) 3C$\mathrm{SiC}$ and $6 \mathrm{H}-\mathrm{SiC}$ NWs with constant cross-sections by the nonequilibrium molecular dynamics (NEMD) method [15]. Bamboo-like SiC NWs were synthesized by a vapor-liquidsolid mechanism and chemical vapor deposition [16-23]. Termentzidis et al. predicated the thermal conductivities of $3 \mathrm{C}-\mathrm{SiC}$ and $2 \mathrm{H}-\mathrm{SiC}$ NWs with constant and variable crosssections via the NEMD method [24], in which the physical model was similar to that of bamboo-like NWs. Zianni appraised the impact of diameter modulation on the thermal conductivity reduction in core-shell silicon nanowires [25]. By a kinetic theory model, Zianni et al. [26-28] investigated transport properties of phonon and electron in diametermodulated nanowires and pointed out the amount of disorder which suppresses thermal conductance deeply according to phonon transmission coefficients.

Approaches to investigate nanoscale heat conduction generally fall into two main categories: analytic and numerical methods. Analytic methods incorporate the Boltzmann transport equation (BTE), Green's theorem, and so on. The most widely used numerical methods are the NEMD and 
MC methods. In the MD method, macroscopic properties can be obtained by calculating the transmission function of every atom. However, if the system contains a large number of atoms, the computations will be immensely expensive. Furthermore, when the simulated temperature is lower than the Debye temperature, the BTE will be invalid for the exchange between classical statistics and quantum statistics, and the results should be revised by quantum corrections. In contrast to the MD method, the MC method treats atomic thermal vibrations as quantum particles and regards atomic thermal interactions as phonon-phonon scattering. Since the calculation of the transmission function is not required in MC simulations, the computational complexity is reduced to a moderate extent. The transmission parameters and distributions of phonons can be gained statistically without quantum corrections at low temperatures.

Based on the Debye model, Peterson first applied the MC method to solve thermal transport in a one-dimensional model by simplifying the phonon group velocities and polarization [29]. Most later MC methods were derived from Peterson's work and extended its computational accuracy and geometric complexity [30-37]. Using realistic phonon spectra, the accuracy of the MC method can be improved significantly [38-43]. In particular, sophisticated nanostructures have attracted more interest for studying the effects of phonon ballistic transport $[44,45]$ and interfacial scattering [46]. Through MC simulations, researchers proposed that the surface roughness is the main factor leading to ultralow and amorphous-limit thermal conductivity [47, 48]. Bong and Wong studied the impact of anisotropy scattering using the MC method and suggested that forward scattering has a more obvious effect on the thermal conductivity than backward scattering [49]. Rickman et al. studied thermal transport across the grain boundary and suggested that one can engineer thermodynamic and transport properties in materials by inducing interfacial layering transitions via changes in temperature or pressure [50].

\section{Monte Carlo Method of Phonon Transport}

In the presented work, the entire MC simulation process is composed of four steps: initialization, drift, scattering, and statistics. For the initialization, the number of phonons with angular frequency $\omega$ at a given temperature $T$ can be calculated by the Bose-Einstein distribution:

$$
n_{\omega}=\frac{1}{\left(e^{\hbar \omega / K_{B} T}-1\right)},
$$

where $\hbar$ denotes the Planck constant and $K_{B}$ represents the Boltzmann constant.

$$
\begin{aligned}
N= & \sum_{i=1}^{1000} n\left(\omega_{i}, \mathrm{LA}\right) D\left(\omega_{i}, \mathrm{LA}\right) \Delta \omega_{i} V \\
& +2 \sum_{i=1}^{1000} n\left(\omega_{i}, \mathrm{TA}\right) D\left(\omega_{i}, \mathrm{TA}\right) \Delta \omega_{i} V
\end{aligned}
$$

where $N$ is the total number of phonons in volume $V$. The phonon spectra are divided into 1000 equal-sized intervals.
The first term on the right-hand side of (2) is the total number of transverse acoustic (TA) phonons, and the second term represents the number of longitudinal acoustic (LA) phonons. $D\left(\omega_{i}, \mathrm{LA}\right)$ and $D\left(\omega_{i}, \mathrm{TA}\right)$ denote the DOS of the LA phonons and TA phonons, respectively. $\Delta \omega_{i}$ is the $i$ th frequency interval. The phonon group velocity $v_{i}$ can be obtained by

$$
v_{i}=\nabla_{k i} \omega_{i}
$$

The temperatures of each cell can be counted by their energies, and this energy can be obtained by

$$
E=\sum_{p} \sum_{i}^{1000}\left(n\left(\omega_{i}, p\right)+\frac{1}{2}\right) \hbar \omega_{i} D\left(\omega_{i}, p\right)
$$

where $E$ is the material volumic energy and $p$ represents the phonon polarization.

After initialization, every phonon has a drifting time step $\Delta t$. $\Delta t$ should be less than the average relaxation time for a phonon to avoid missing phonon scattering events. In this work, $\Delta t$ is set at 1.0 ps. Based on Matthiessen's formula, the average relaxation time $\tau$ can be calculated by

$$
\frac{1}{\tau}=\frac{1}{\tau_{I}}+\frac{1}{\tau_{N}}+\frac{1}{\tau_{U}}+\frac{1}{\tau_{B}},
$$

where $\tau_{I}$ is the phonon impurity scattering relaxation time [31]. $\tau_{N}$ denotes the normal scattering relaxation time, and $\tau_{U}$ is the Umklapp scattering relaxation time. $\tau_{B}$ is the boundary scattering relaxation time. Given the value of $\tau$, the average scattering probability $p$ can be calculated from

$$
p=1-\exp \left(-\frac{\Delta t}{\tau}\right)
$$

Whether a scattering event occurs should be judged by comparing the value of $p$ and a generated random number. In this work, $\tau_{B}$ is not included in (6). The conventional approach to solve phonon boundary scattering is composed of an indirect method and a direct method. Introduced in Ziman's work [51] and developed by scholars [52, 53], the indirect method has a simplified $\tau_{B}$, which assumes that the lateral surfaces of the simulated structure are flat. In the direct method, boundary scattering only depends on whether its position exceeds the boundary. In our study, the direct method is adopted. If a boundary scattering event occurs, the phonon will undergo diffuse reflection. The thermal flux and thermal gradient can be gathered by the synchronous ensemble method at each time $t_{i}$ in Figure 1 . The vertical solid lines denote the time point $t_{i}$. The interval time between two adjacent dashed lines is time step $\Delta t$. The distance between two adjacent solid lines is $t_{i}-t_{i-1}=n \times \Delta t$, where $n$ is set to $10^{2}-10^{3}$ in the simulations. To simplify the explanations in Figure 1, $n$ is set to 4 . The transverse lines represent the time course of phonon 1 and phonon 2 . Solid circles denote impurity scattering or U scattering, and hollow circles denote boundary scattering.

At each time point $t_{i}$, all sample phonons should be relabeled, and their velocities and polarization should be 


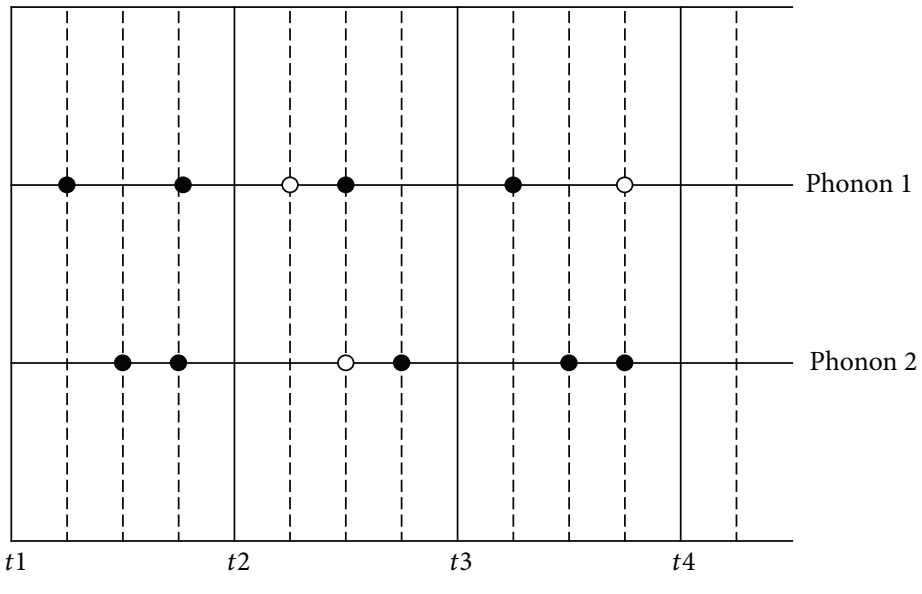

FIGURE 1: The schematic diagram of synchronous ensemble method.
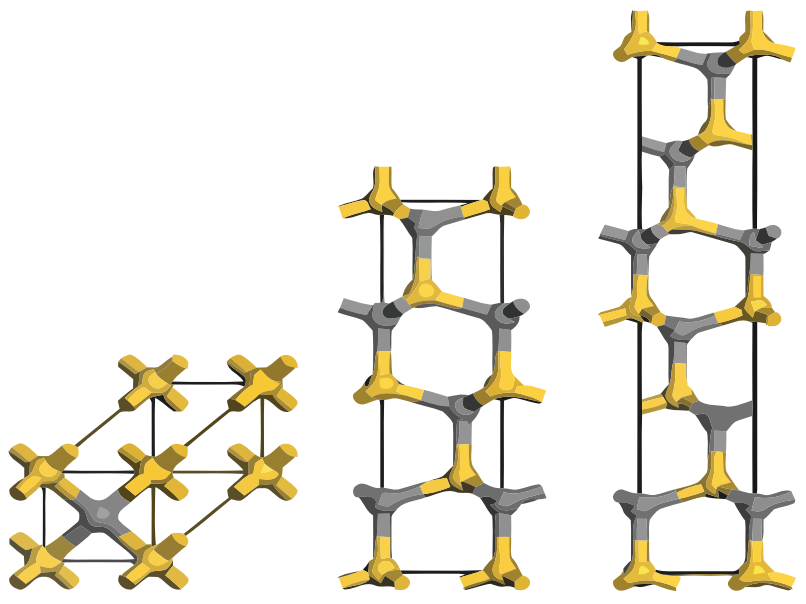

Figure 2: Schematics of 3C-SiC, $4 \mathrm{H}-\mathrm{SiC}$, and $6 \mathrm{H}-\mathrm{SiC}$.

inherited. The energy variation of each cell needs be counted to calculate the thermal flux. The energy-temperature list may be calculated from (4) in advance. Using the bisection method, the temperature of each cell can be determined by the energy-temperature list. Given the thermal flux and temperature gradient, thermal conductivities can be worked out by Fourier's law.

\section{Models for Phonon Transport in Bamboo-Like NWs}

As shown in Figure 2, 3C-SiC has a zinc blende crystal structure with an $\mathrm{ABC}$ stacking sequence, while $4 \mathrm{H}-\mathrm{SiC}$ and $6 \mathrm{H}-\mathrm{SiC}$ have hexagonal crystal structures with $\mathrm{ABCB}$ and $\mathrm{ABCACB}$ stacking sequences. In Figure 3, hollow circles in the bamboo-like schematic drawing denote phonons distributed in the bamboo-like NWs, and the right-top inset is a scanning electron microscopy (SEM) image [21]. The thermal flux flows along the positive direction, indicated by the arrow symbol, from the hot part to the cold part. If we swap the positions of the hot part and cold part,

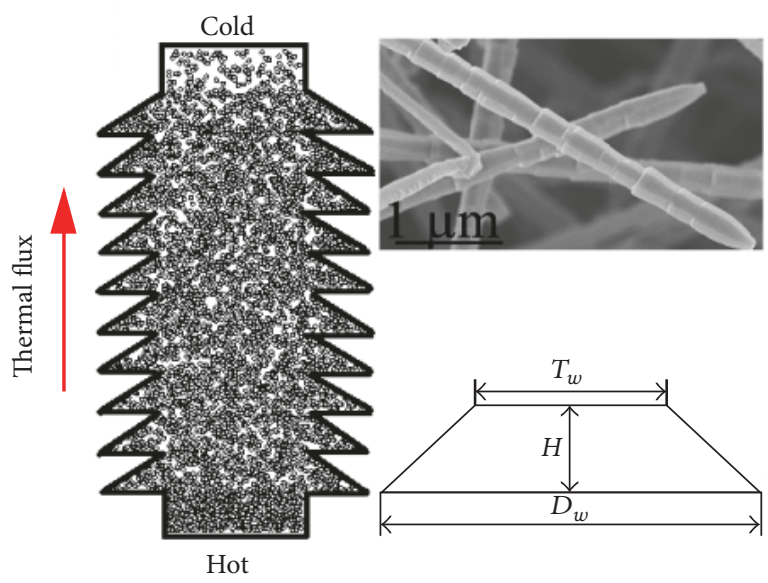

FIgure 3: Physical model of 3C-SiC NW; hollow circles denote phonons. Top-right inset is a SEM image of 3C-SiC NW; down-right inset is a schematic of one bamboo joint.

the thermal flux will flow along the negative direction. The right-bottom inset is a bamboo joint schematic drawing confined by three parameters: $T_{w}, H$ and $D_{w}$. $T_{w}$ represents the bamboo joint's top width, $H$ is its height, and $D_{w}$ denotes its bottom width. $\left(D_{w}-T_{w}\right) / T_{w}$ is defined as the aspect ratio to modulate the shape of the bamboo joint. In simulations, given $T_{w}$, we can change the values of $D_{w}$ and $H$ to investigate the phenomena of phonon diffuse boundary scattering and thermal rectification effects.

In this work, three kinds of phonon scattering are considered: impurity scattering, U scattering, and boundary scattering. To consider impurity scattering, we use the same equation as [26] to calculate its relaxation rates. Since $U$ scattering obeys the conservation of energy and momentum, it can be neglected in the simulations. The combined phonon relaxation time $\tau$ for impurity scattering and $U$ scattering can be given by Matthiessen's formula, as in (5), and the possibility of boundary scattering is only dependent on its locations in the simulated physical model. 


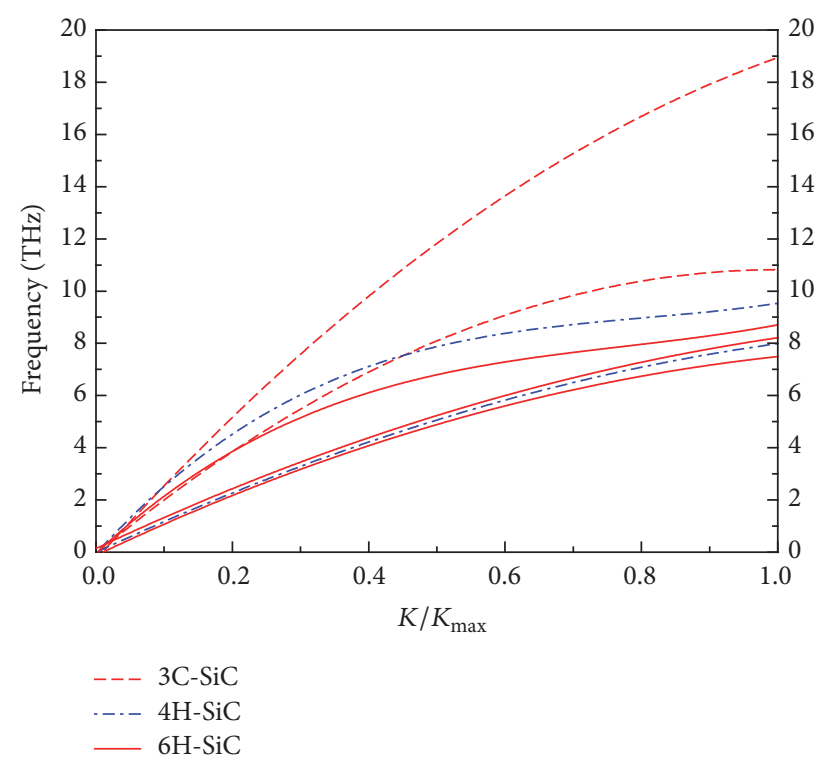

Figure 4: Acoustic spectra of 3C-SiC, $4 \mathrm{H}-\mathrm{SiC}$, and $6 \mathrm{H}-\mathrm{SiC}$.

3.1. Phonon U Scattering Rate for $3 \mathrm{C}-\mathrm{SiC}, 4 \mathrm{H}-\mathrm{SiC}$, and $6 \mathrm{H}-\mathrm{SiC}$. From a theoretical point of view, thermal vibrations are often quantized as phonons, obeying the Boltzmann distribution. The phonon energy can be obtained from the spectra of 3C$\mathrm{SiC}, 4 \mathrm{H}-\mathrm{SiC}$, and $6 \mathrm{H}-\mathrm{SiC}$, shown in Figure 4. Since optical phonons have much lower velocities than acoustic ones, we neglect the optical branches, following the previous literature $[49,54]$, in our simulations. Compared with $4 \mathrm{H}-\mathrm{SiC}$ and $6 \mathrm{H}-$ $\mathrm{SiC}, 3 \mathrm{C}-\mathrm{SiC}$ has a higher acoustic cut-off frequency, as shown in Figure $4 . K_{\max }$ of $3 \mathrm{C}-\mathrm{SiC}$ is $2 \pi / a$ along the [001] direction, while $4 \mathrm{H}-\mathrm{SiC}$ and $6 \mathrm{H}-\mathrm{SiC}$ have the same expressions $K_{\max }=$ $2 \pi / a / 3^{1 / 2}$ along the [010] direction, where $a$ is the length of the axial vector [55].

When a phonon undergoes a $U$ scattering event with two other phonons, their energies and wave vectors will be changed, as shown in the first Brillouin zone in Figure 5. For example, T1 is one of the degenerate acoustic transverse branches of $3 \mathrm{C}-\mathrm{SiC}$, and $\mathrm{T} 2$ is the translational curve of $\mathrm{T} 1 . \mathrm{L}$ is the acoustic longitudinal branch, and $\mathrm{L}^{\prime}$ is the mirroring curve of $\mathrm{L}$ at the symmetry axis of $K=1 . \mathrm{P} 2$ and $\mathrm{P} 3$ are intersection points of curve T2 and $\mathrm{L}^{\prime}$. Point P2 belongs to curve T2, while $\mathrm{P} 3$ belongs to T3. P1 and P2 represent two acoustic phonons with $\omega_{1}$ and $\omega_{2}$, while $\mathrm{P} 3$ represents a longitudinal phonon with $\omega_{3}$. They satisfy the $U$ scattering requirement $\omega_{1}+\omega_{2}=\omega_{3}$ and $k_{1}+k_{2}=G+k_{3}$, where $G$ denotes the reciprocal lattice vector. There are several types of U scattering events: $L+T \leftrightarrow L, T+T \leftrightarrow L$, and $T+L \leftrightarrow L$. The expressions of the phonon $\mathrm{U}$ scattering relaxation rate for the former two $\mathrm{U}$ scattering events are given as

$$
\frac{1}{\tau_{U}} \approx \frac{2 \gamma^{2} \hbar}{3 \pi \rho \nu_{\mathrm{ph}}^{2} \nu_{g}} \omega_{i} \omega_{T}\left(\omega_{i}+\omega_{T}\right) r_{c}^{2} \exp \left(-\frac{\hbar \omega_{T}}{k_{B} T}\right)
$$

where $\gamma$ is the Grüneisen parameter, $\gamma=0.76$ for $3 \mathrm{C}-\mathrm{SiC}$ [56], and $\gamma=1.23$ for $6 \mathrm{H}-\mathrm{SiC}$ and $4 \mathrm{H}-\mathrm{SiC}$ [57]. $\hbar$ is Planck's constant, $k_{B}$ is the Boltzmann constant, and $\rho$ is the density

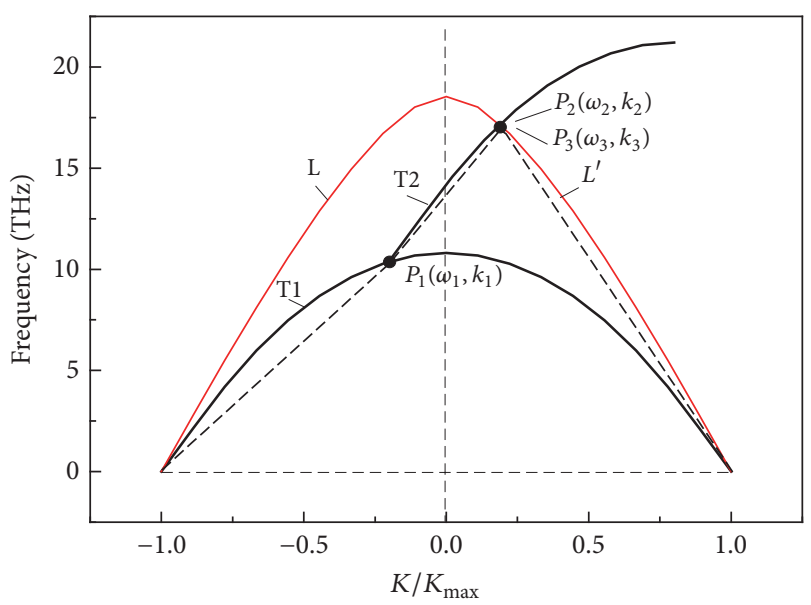

FIGURE 5: The process of Umklapp scattering for 3C-SiC in the first Brillouin zone.

of $\mathrm{SiC} . \mathrm{T}$ is the local temperature. $\nu_{\mathrm{ph}}$ is the phase velocity, and $\nu_{g}$ is the group velocity. $r_{c}$ is the optimum radius of the integral surface in the process of $U$ scattering [58]. For the event $T+L \leftrightarrow L$, (8) can be employed to calculate $\tau_{U}$ :

$$
\begin{aligned}
& \frac{1}{\tau_{U}} \\
& \quad \approx \frac{\gamma^{2} \hbar}{3 \pi \rho v_{\mathrm{ph}}^{2} \nu_{g}} \omega_{i}\left(\omega_{L}-\omega_{i}\right) \omega_{L} r_{c}^{2} \exp \left[-\frac{\hbar\left(\omega_{L}-\omega_{i}\right)}{k_{B} T}\right] .
\end{aligned}
$$

Figure 6 shows that the relaxation rate for $T+T \leftrightarrow L$ is much larger than those for $T+L \leftrightarrow L$ and $L+T \leftrightarrow L$, since the number of transverse acoustic phonons participating in $U$ scattering is much larger than that of longitudinal acoustic phonons, according to the Bose-Einstein distribution. Figure 7 shows the $\mathrm{U}$ scattering combined relaxation rates for $3 \mathrm{C}-\mathrm{SiC}, 4 \mathrm{H}-$ $\mathrm{SiC}$, and $6 \mathrm{H}-\mathrm{SiC}$. Below $200 \mathrm{~K}$, the $\mathrm{U}$ scattering relaxation rates decrease sharply with increasing temperature. Above $400 \mathrm{~K}$, the $\mathrm{U}$ scattering relaxation rates for the three $\mathrm{SiC}$ polytypes converge to the orders of $10^{-11}-10^{-12}$ gradually. $3 \mathrm{C}$ $\mathrm{SiC}$ has the largest $\mathrm{U}$ scattering relaxation rate, while $4 \mathrm{H}$ $\mathrm{SiC}$ has a closer value to that of $6 \mathrm{H}-\mathrm{SiC}$ above $100 \mathrm{~K}$. From this, it is can be speculated that $6 \mathrm{H}-\mathrm{SiC}$ has a larger thermal conductivity than $3 \mathrm{C}-\mathrm{SiC}$ or $4 \mathrm{H}-\mathrm{SiC}$, which is in accordance with experiments [59].

3.2. Phonon Boundary Scattering in Bamboo-Like NWs. While transporting through bamboo-like NWs, a phonon may encounter a large number of boundary scattering events. The border of the bamboo-like NWs should be formulized first. If a phonon's coordinates surpass the border, it will be reflected diffusely by the boundary because of lattice defects and surface stresses. The direction of its velocity will be changed along the border randomly following equation:

$$
\left[V_{i x}, V_{i y}\right] \times P=V_{i}\left[\cos \left(\pi R_{1}\right), \sin \left(\pi R_{2}\right)\right] \times P,
$$

where $V_{i x}$ and $V_{i y}$ represent the components of the velocity along the $x$-axis and $y$-axis. $R_{1}$ and $R_{2}$ are generated random 


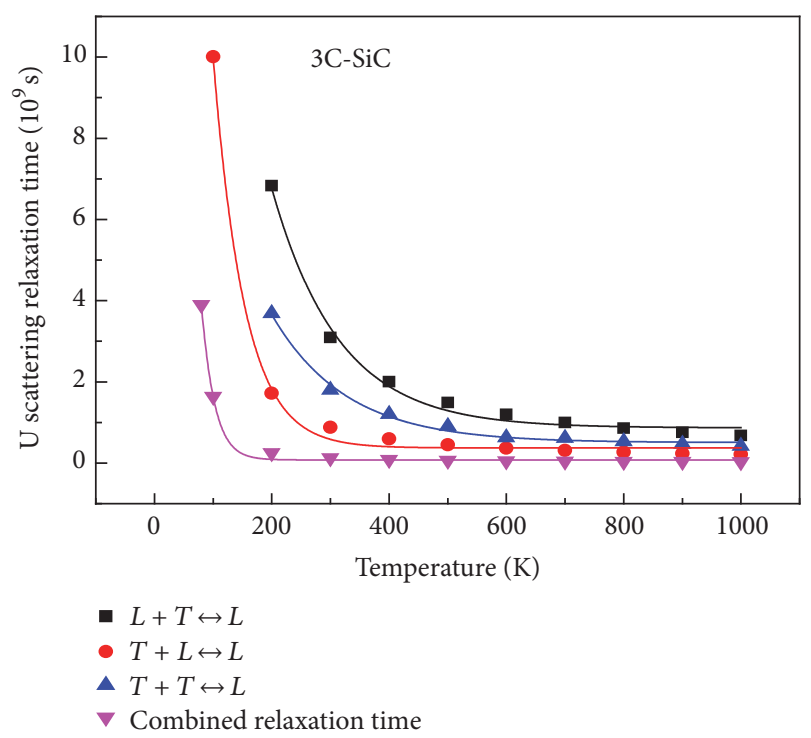

FIGURE 6: U scattering relaxation rates of 3C-SiC against temperatures.

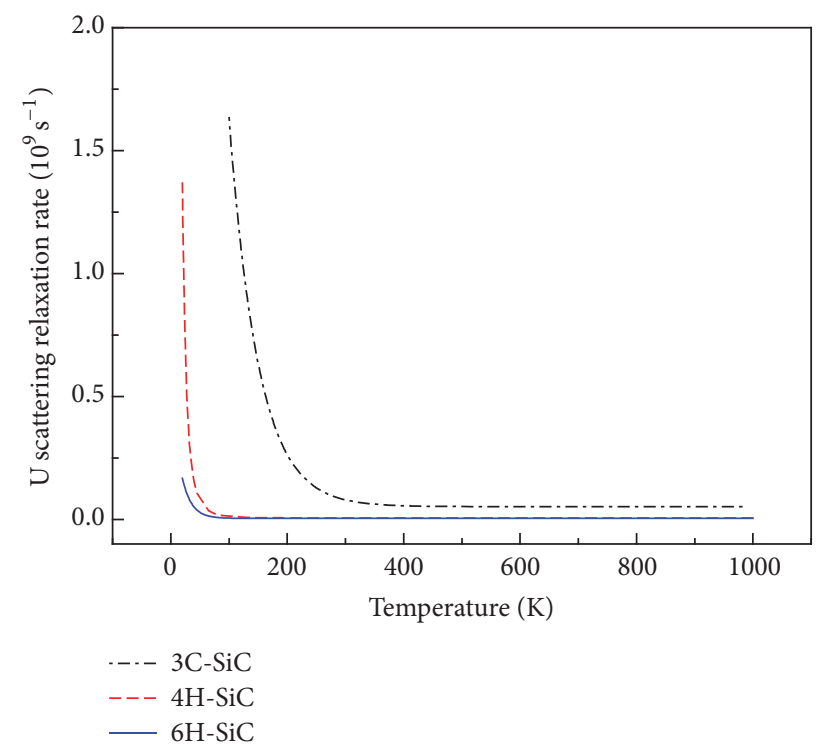

FIGURE 7: U scattering phonon combined relaxation rates of $\mathrm{SiC} 3 \mathrm{C}$ $\mathrm{SiC}, 4 \mathrm{H}-\mathrm{SiC}$, and $6 \mathrm{H}-\mathrm{SiC}$ against temperatures.

numbers. $P$ is a coordinate transformation matrix decided by the equation of the boundary border. A sample phonon's flying trajectories are recorded as hollow circles in Figure 8 for a period of $100 \Delta t$. When the sample phonon knocks at the boundary, it will be reflected diffusely.

\section{Results and Discussion}

To verify our MC model and determine the value of the impurity scattering parameter $\mathrm{Bi}$, the bulk thermal conductivities of the three $\mathrm{SiC}$ polytypes were calculated and are given in Figure 9. The circle symbols, triangle symbols, and rectangle symbols represent the experimental values [60]. The curves

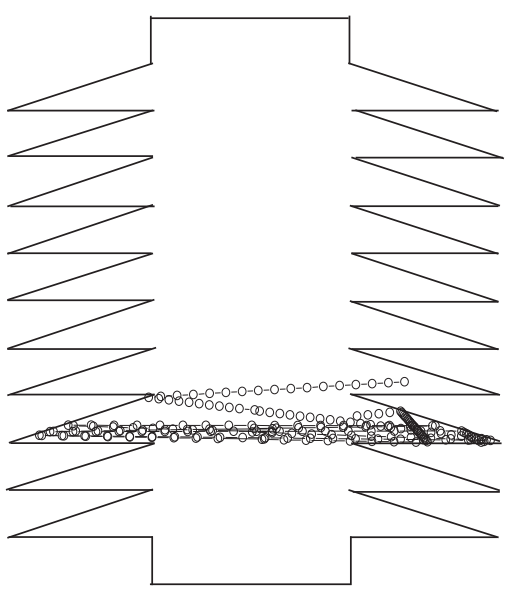

FigURE 8: Schematic of phonon transport trajectories.

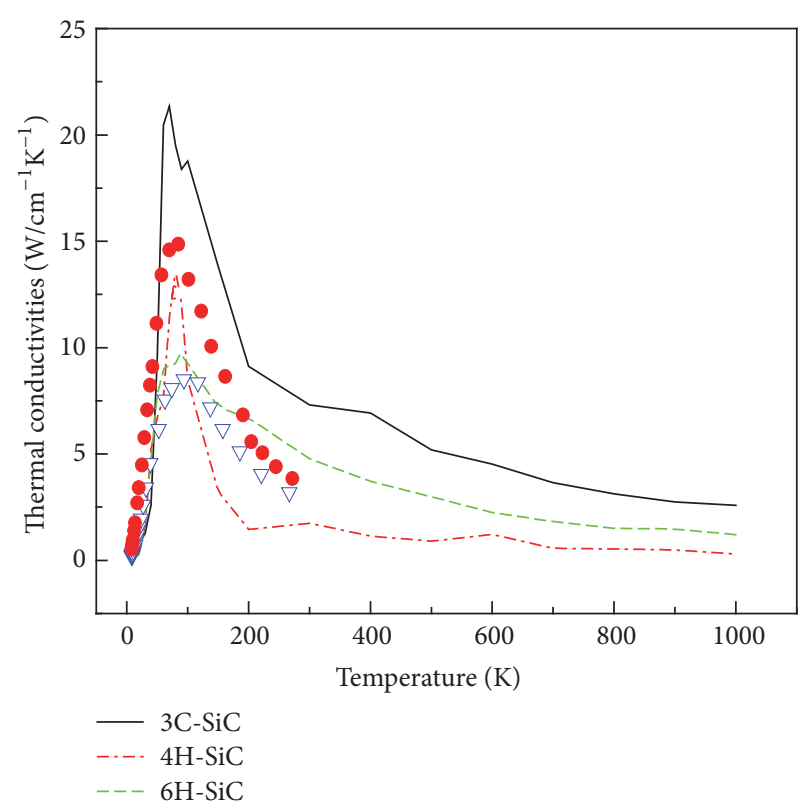

FIGURE 9: Experimental and simulated values of thermal conductivities of $\mathrm{SiC}$ polytypes in bulk.

were obtained by our MC simulations of impurity scattering and $\mathrm{U}$ scattering. Below $100 \mathrm{~K}$, the thermal conductivities fluctuate drastically at low temperatures and reach peak values at $80-100 \mathrm{~K}$. Above $600 \mathrm{~K}$, the thermal conductivities of the three polytypes converge to $0.5-2 \mathrm{~W} / \mathrm{cm} \cdot \mathrm{K}$. In this case, the impurity scattering parameter $\mathrm{Bi}$ is fit to $5 \times 10^{-48}$ from the experiments, which is fixed in our simulations. We employ Fourier's law to calculate the thermal conductivities by the average cross-sectional area of the bamboo junction. As shown in Figure 9, the discrepancies between the experiments and the simulations are considerable. This gap may result from two sources: one is from the approximate value of $\mathrm{Bi}$, which is uncertain in the experiments [60], while the other is perhaps from the Grüneisen parameters in (7) and (8), which are also approximate values in $[56,58]$. Though there are considerable gaps between the simulations and 


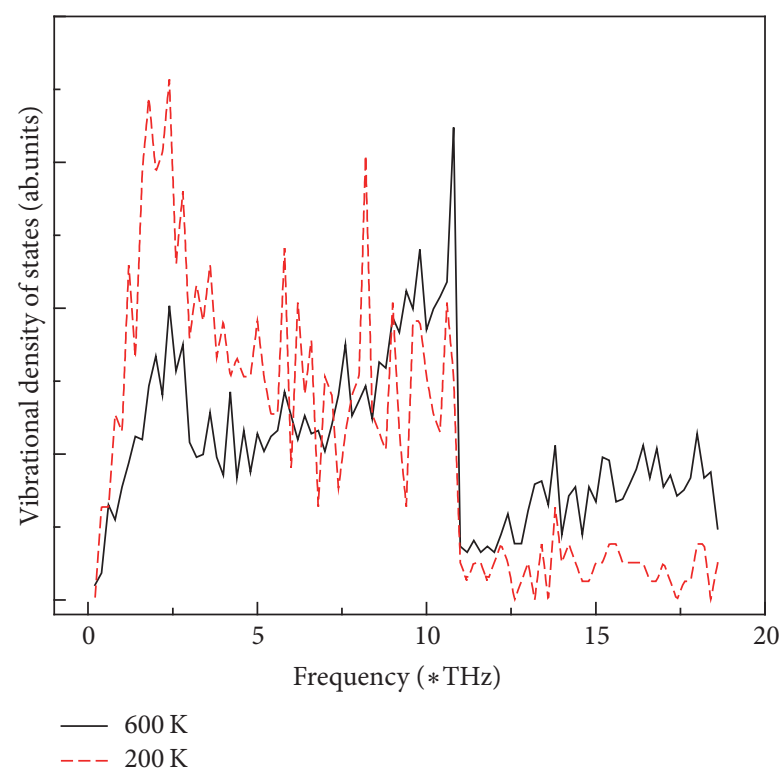

FIgURE 10: DOS of phonon frequency for 3C-SiC.

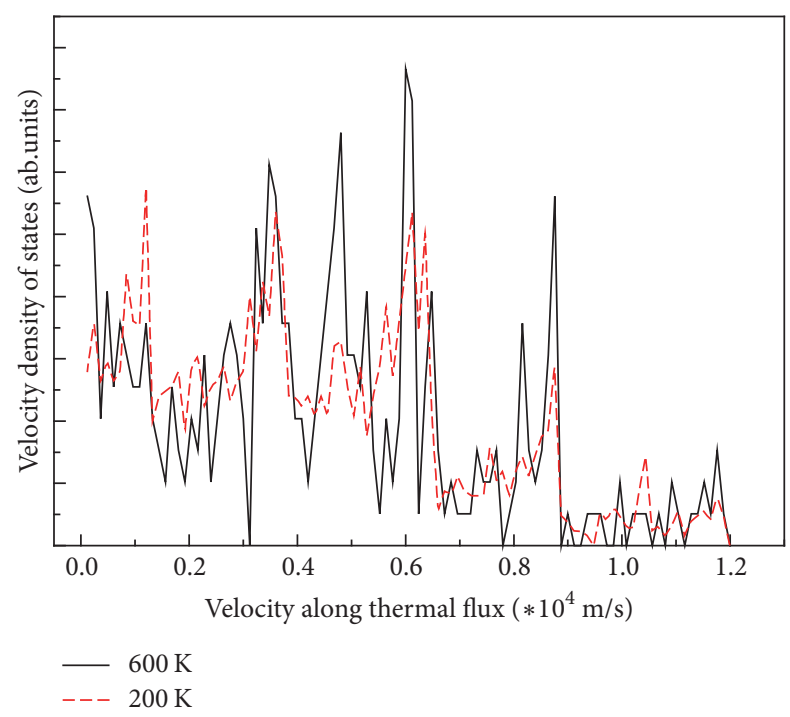

FIGURE 11: DOS of phonon velocity for 3C-SiC.

experiments, the overall trends match well with experiments. To some extent, this MC model is helpful to investigate thermal transport in nanoscale structures qualitatively.

4.1. Density of States (DOS) of Phonon Frequency and Velocity in $3 C$-SiC. From a phonon's DOS, distributions of the phonon frequency and velocity can be obtained directly, which is beneficial to analyze $U$ scattering and boundary scattering in bamboo-like NWs. Figures 10 and 11 show the DOS of the phonon frequency and phonon velocity of 3C$\mathrm{SiC}$ at $200 \mathrm{~K}$ and $600 \mathrm{~K}$. It can be seen in Figure 10 that, with increasing temperature, the ratio of phonons with high frequencies increases. Figure 11 indicates the variation in the phonon group velocity. The average phonon group velocity

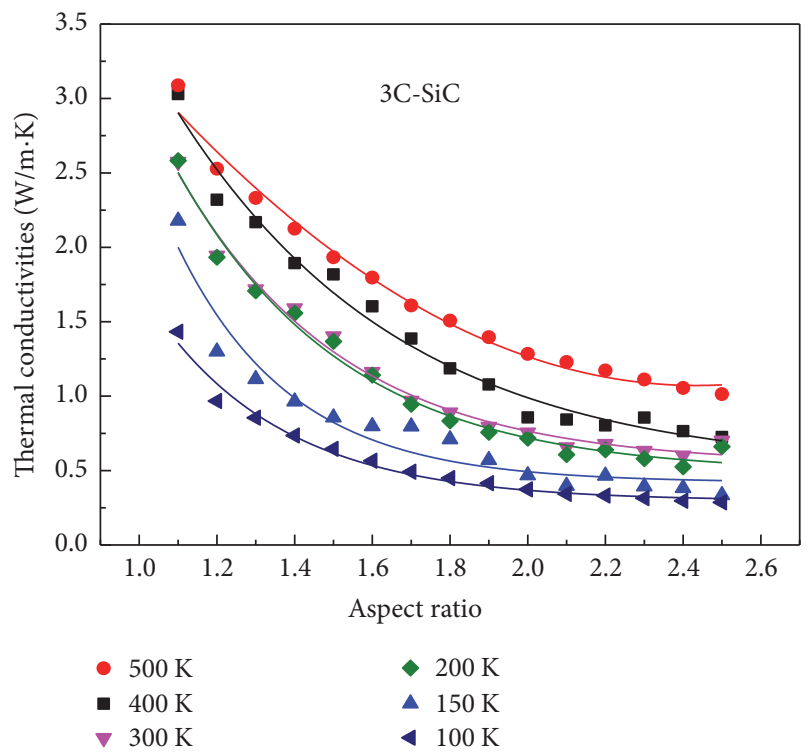

FIgURE 12: Thermal conductivities in positive direction against aspect ratio.

equals $4276.3 \mathrm{~m} / \mathrm{s}$ at $600 \mathrm{~K}$ and equals $4441.5 \mathrm{~m} / \mathrm{s}$ at $200 \mathrm{~K}$, which manifests that a higher temperature leads to a lower phonon group velocity. We also calculated the DOS of the phonon frequency and phonon velocity of $4 \mathrm{H}-\mathrm{SiC}$ and $6 \mathrm{H}$ $\mathrm{SiC}$. The results show that the three $\mathrm{SiC}$ polytypes have similar trends of phonon frequency and group velocity.

4.2. Phonon Transport in Bamboo-Like 3C-SiC NWs. Boundary effects play an important role in nanoscale thermal transport. It is worth researching the thermal conductivities and thermal rectifications of bamboo-like NWs quantitatively. As Figure 12 shows, the thermal conductivities of bamboo-like NWs with different sizes are simulated along the positive direction. $T_{w}$ is fixed at $100 \mathrm{~nm}$, and the thickness of the simulated NWs is set to $50 \mathrm{~nm}$. The thermal conductivities of $3 \mathrm{C}-\mathrm{SiC}$ with different aspect ratios are presented over temperatures from $100 \mathrm{~K}$ to $500 \mathrm{~K}$. Since the number of phonons with high velocities decreases as the temperature rises, the boundary scattering relaxation rate also decreases with increasing temperatures. Hence, in contrast to bulk conditions, the thermal conductivities at high temperatures are larger than those at low temperatures in bamboo-like NWs. Additionally, the thermal conductivity becomes steady and irrelevant at aspect ratios above 2.3. In Figure 13, we investigate the dependence of the thermal conductivity on $H$, with values of $100 \mathrm{~nm}, 150 \mathrm{~nm}$, and $200 \mathrm{~nm}$. When increasing $H$ from $150 \mathrm{~nm}$ to $200 \mathrm{~nm}$, the gap between the thermal conductivities is smaller than that when going from $100 \mathrm{~nm}$ to $150 \mathrm{~nm}$. To further study boundary scattering, we trace 5000 sample phonons and calculate their average boundary relaxation time, which is shown in the inset of Figure 13. The phonon boundary scattering relaxation time for $H=150 \mathrm{~nm}$ is very close to that of $H=200 \mathrm{~nm}$ and apparently larger than that of $H=100 \mathrm{~nm}$, which affirms exactly the effect of boundary scattering. 


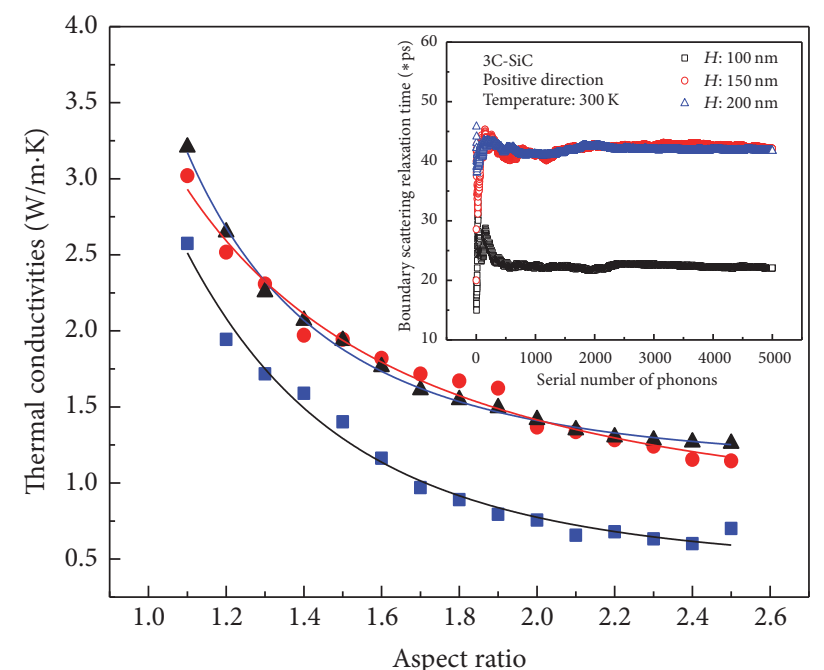

\ $H: 200 \mathrm{~nm}$

- $H: 150 \mathrm{~nm}$

- $H: 100 \mathrm{~nm}$

FIgURE 13: Thermal conductivities in positive direction against $H$. Inset: boundary scattering relaxation time.

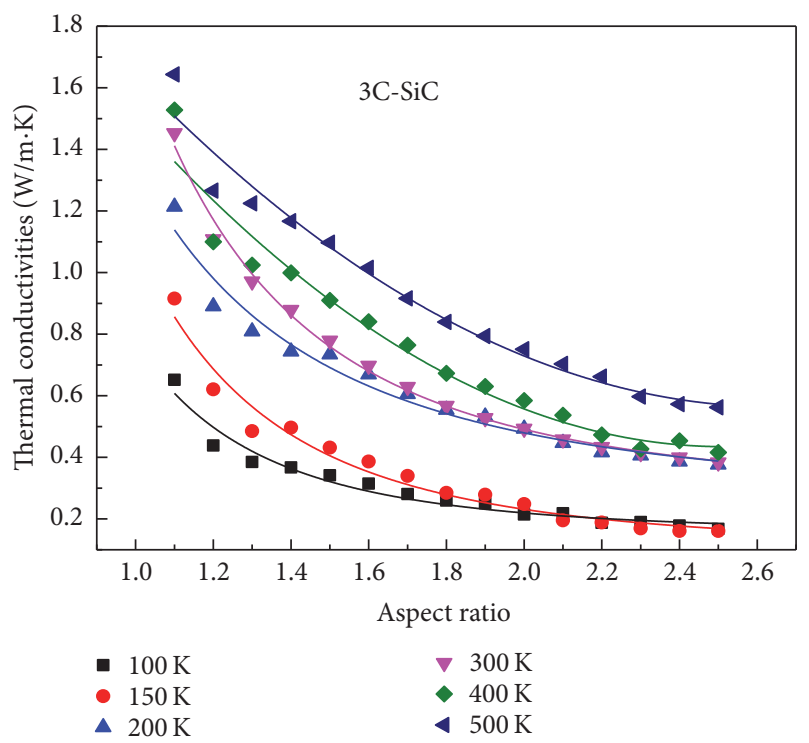

FIGURE 14: Thermal conductivities in negative direction against aspect ratio.

Moreover, the thermal conductivities in the negative direction are investigated in Figures 14 and 15. As shown in Figure 14, the thermal conductivities increase with temperature and reach a steady state when the aspect ratio exceeds 2.4. The thermal conductivities in the negative direction are significantly lower than those in the positive direction. In Figure 15, the bamboo-like NW with $H=100 \mathrm{~nm}$ has the lowest thermal conductivities, as seen in Figure 13. The gap between NWs with $H=150 \mathrm{~nm}$ and $H=200 \mathrm{~nm}$ is larger than that in the positive direction. The inset figures in Figures 13 and 15 show that the boundary scattering relaxation time in the negative direction is shorter than that in the positive

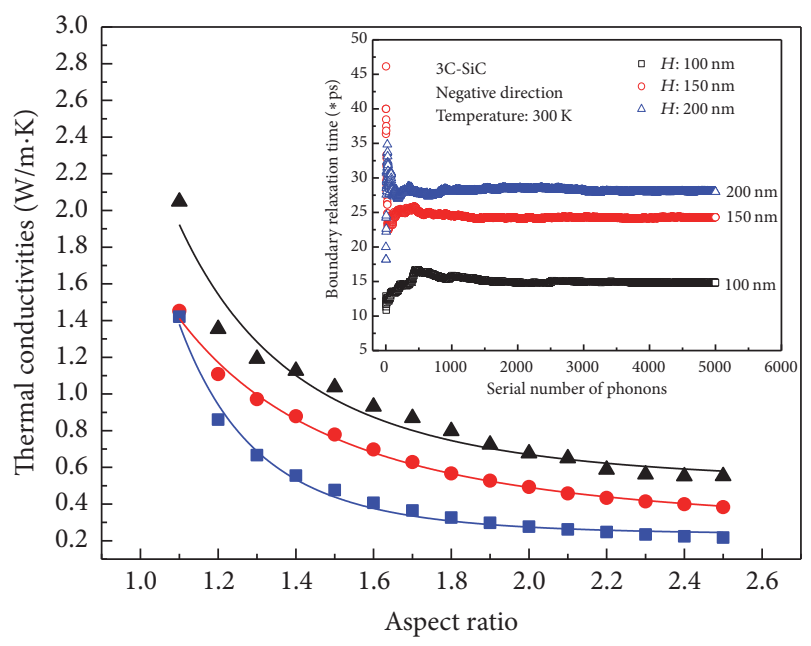

A $H: 200 \mathrm{~nm}$

- $H: 150 \mathrm{~nm}$

- $H: 100 \mathrm{~nm}$

FIGURE 15: Thermal conductivities in negative direction against aspect ratio.

direction, which gives rise to a higher possibility for boundary scattering.

Thermal conductivity trends to maintain steady. The insets also indicate the discrepancies of boundary relaxation time between different aspect ratios decrease with the increasing value of aspect ratios. That is, the contribution of phonon boundary scattering to thermal resistances is restricted by the aspect ratio. Moreover, thermal conductivities of $4 \mathrm{H}-\mathrm{SiC}$ and $6 \mathrm{H}-\mathrm{SiC}$ in positive direction and negative direction are simulated, and the calculated results are used to thermal rectification in the next section.

4.3. Thermal Rectification of Bamboo-Like 3C-SiC, 4H-SiC, and $6 \mathrm{H}-\mathrm{SiC} \mathrm{NWs}$. The thermal rectification effect is similar to the regime of the electronic diode, which exists in bamboolike SiC NWs because of the different boundary scattering rates in the positive and negative directions. The efficiency of thermal rectification $\eta$ can be obtained from the following equation $[61,62]$ :

$$
\eta=\frac{\left(\lambda_{p}-\lambda_{n}\right)}{\lambda_{p}},
$$

where $\lambda_{p}$ and $\lambda_{n}$ represent the thermal conductivities in the positive and negative directions, respectively. Figure 16 shows the thermal conductivities and rectification efficiencies $\eta$ in the negative and positive directions at $300 \mathrm{~K}$. With increasing aspect ratio, the thermal conductivities in the positive and negative models for $3 \mathrm{C}-\mathrm{SiC}$ decrease first and then converge to $0.3 \mathrm{~W} / \mathrm{m} \cdot \mathrm{K} . \eta$ increases from zero with increasing aspect ratio below 1.1 and then decreases to zero with a further increase in the aspect ratio. The trends are caused by the dependency of phonon boundary scattering on the aspect ratio. As $D_{w}$ approaches $T_{w}$, the aspect ratio approaches zero, which would represent a constant cross-section nanowire. 


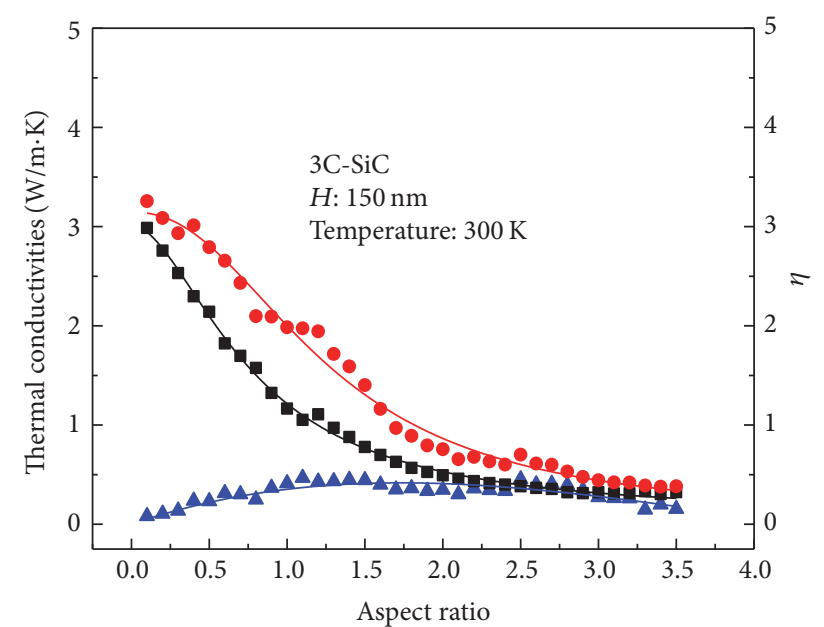

- Positive

- Negative

- Thermal rectification efficiency

FIGURE 16: Thermal rectification of 3C-SiC against aspect ratio.

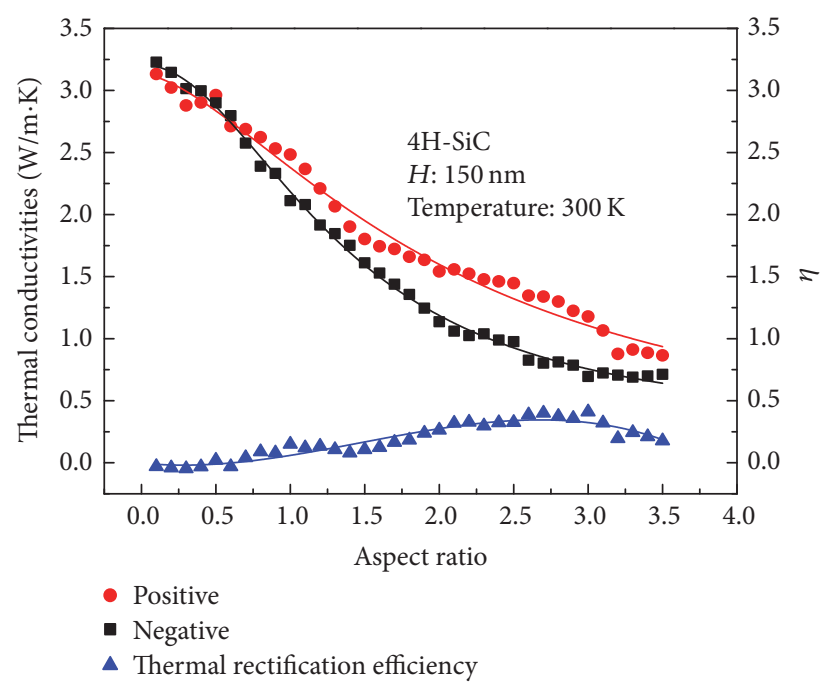

FIGURE 17: Thermal conductivity and rectification efficiency of $4 \mathrm{H}-$ $\mathrm{SiC}$ against variable aspect ratio.

In this case, the rectification efficiency should also approach zero, and the resulting thermal conductivities in the forward and reverse directions can be compared to constant crosssection values. In addition, as the aspect ratio approaches infinity, the bamboo features also diminish, which also may result in near-zero rectification efficiencies.

In Figure 17, we also calculate $\lambda_{p}, \lambda_{n}$, and $\eta$ for $4 \mathrm{H}$ SiC. Compared with $3 \mathrm{C}-\mathrm{SiC}, 4 \mathrm{H}-\mathrm{SiC}$ has higher $\lambda_{p}$ and $\lambda_{n}$, which is consistent with the deduction from Figure 7 . To study the contributions of boundary scattering, the phonon boundary relaxation time for $3 \mathrm{C}-\mathrm{SiC}, 4 \mathrm{H}-\mathrm{SiC}$, and $6 \mathrm{H}-\mathrm{SiC}$ was calculated and is given in Figure 18. The phonon boundary scattering relaxation time (inversely proportional to the boundary scattering relaxation rate) for $3 \mathrm{C}-\mathrm{SiC}$ is shorter

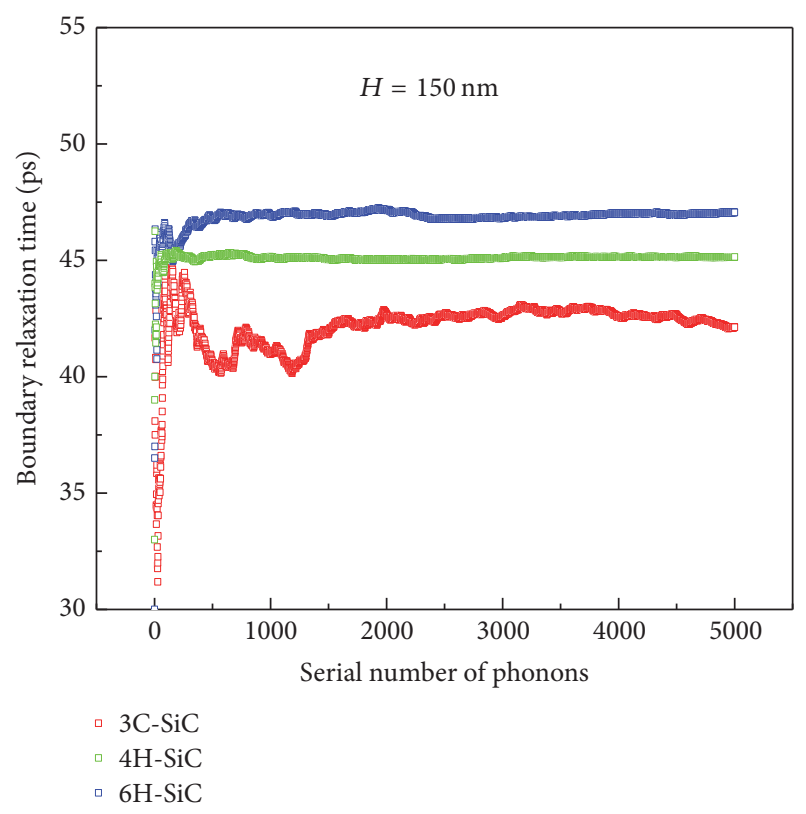

FIGURE 18: Phonon boundary scattering relaxation time in positive direction.

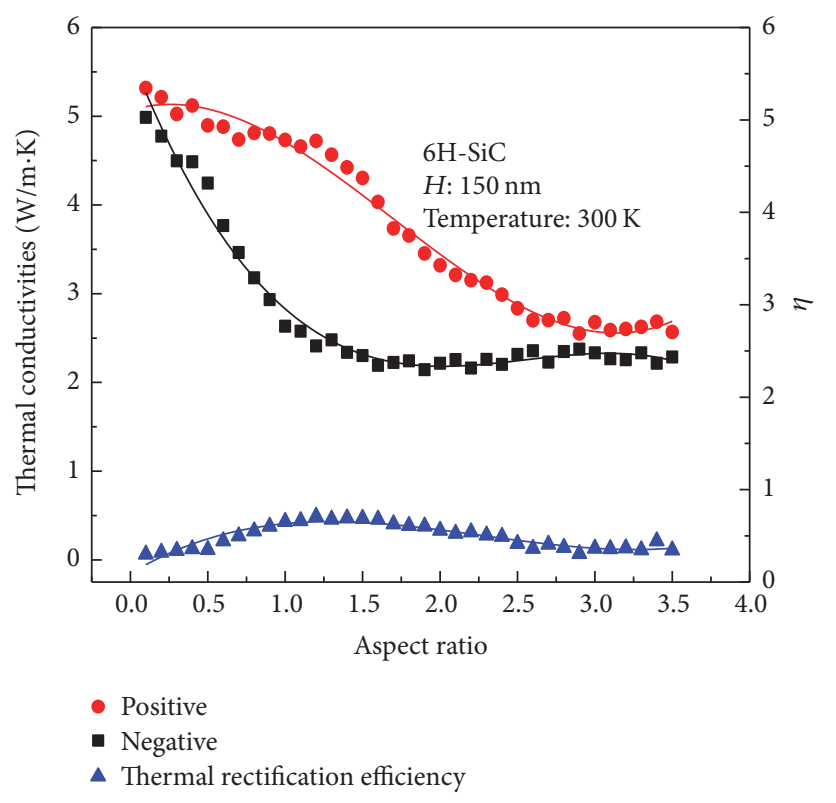

FIGURE 19: Thermal conductivity and rectification efficiency of $6 \mathrm{H}-$ $\mathrm{SiC}$ against variable aspect ratio.

than that for $4 \mathrm{H}-\mathrm{SiC}$, resulting in a lower thermal conductivity. Namely, thermal resistances arising from boundary scattering in the bamboo-like NWs are more predominant for $3 \mathrm{C}-\mathrm{SiC}$ than for $4 \mathrm{H}-\mathrm{SiC}$ or $6 \mathrm{H}-\mathrm{SiC}$. The reason mainly relies on the differences between their phonon spectra, which leads to distinctive phonon group velocities and polarization.

In comparison to $3 \mathrm{C}-\mathrm{SiC}$ and $4 \mathrm{H}-\mathrm{SiC}, 6 \mathrm{H}-\mathrm{SiC}$ has the highest $\lambda_{p}$ and $\lambda_{n}$, as shown in Figure 19, due to the lowest $\mathrm{U}$ scattering rate and lowest boundary scattering rate. It can also be seen that the three studied $\mathrm{SiC}$ polytypes have 
different peak values of $\eta$, and the highest $\eta$ is less than 0.5 . Thus, by means of modulating the shape of the bamboolike NWs, the thermal conductivities can be dropped by 200-500 times, compared with the bulk values. However, when the aspect ratio increases or decreases away from the value corresponding to the peak $\eta$, the thermal rectification efficiency becomes ambiguous, and the trend of $\eta$ is shaped as a parabola going downwards.

In the previous works $[9,10,24,61]$, most of the authors have similar predictions that thermal conductivity decreases with the increasing aspect ratio. Different from their opinions, we suggest that thermal conductivities in positive direction and negative position do not increase with the aspect ratio at all times. When the aspect ratio increases above 2.4 , thermal conductivity trends to maintain steady. The insets of Figures 13 and 15 indicate the discrepancy of boundary relaxation time between different aspect ratios decrease with the increasing value of aspect ratios. That is, the contributions of phonon boundary scattering to thermal resistances are restricted by the aspect ratio.

\section{Conclusion}

We have presented MC investigations of bamboo-like SiC NWs for $3 \mathrm{C}-\mathrm{SiC}, 4 \mathrm{H}-\mathrm{SiC}$, and $6 \mathrm{H}-\mathrm{SiC}$ on phonon transport. Particularly, phonon $\mathrm{U}$ scattering relaxation rates and boundary scattering relaxation time are calculated separately in this work, providing qualitative theoretical supports to future MC studies. It is observed the special shape of the bamboo-like boundary plays an essential role in reducing the thermal conductivities as previous reports [25-28]. Particularly at low temperatures, the thermal conductivities are suppressed distinctly. Thermal conductivities in the positive direction are higher than those in the negative direction, which is because the boundary scattering relaxation rate in the positive direction is lower than that in the negative direction. Increasing the aspect ratio from 0.1 to 3.5 , the rectification efficiency increases from zero at first and then decreases to zero with further increase in the aspect ratio, which demonstrates that the geometric shape of the bamboo-like junction has limited effect on tailoring the thermal rectification efficiency.

\section{Conflicts of Interest}

The author declares that they have no conflicts of interest.

\section{Acknowledgments}

Z. Wang would like to acknowledge the financial support from the National Natural Science Foundation of China (51106043 and 51373048) and the basic research project of Henan Provincial Department of Education Science and Technology (13A470176 and 2014GGJS-059).

\section{References}

[1] N. Papanikolaou, "Nonequilibrium radiation dosimetry," Journal of Physics: Condensed Matter, vol. 20, Article ID 135201, 2008.
[2] D.-H. Wang, D. Xu, Q. Wang et al., "Periodically twinned SiC nanowires," Nanotechnology, vol. 19, no. 21, Article ID 215602, 2008.

[3] J. W. Lyver IV and E. Blaisten-Barojas, "Lattice thermal conductivity in $\mathrm{SiC}$ nanotubes, nanowires and nanofilaments: A molecular dynamics study," Journal of Computational and Theoretical Nanoscience, vol. 8, no. 4, pp. 529-534, 2011.

[4] H. Li, Z. He, Y. Chu, L. Qi, and Q. Fu, "Large-scale synthesis, growth mechanism, and photoluminescence of 3C-SiC nanobelts," Materials Letters, vol. 109, pp. 275-278, 2013.

[5] X. Zhang, M. Hu, K. P. Giapis, and D. Poulikakos, "Schemes for and mechanisms of reduction in thermal conductivity in nanostructured thermoelectrics," Journal of Heat Transfer, vol. 134, no. 10, Article ID 102402, 2012.

[6] L. Zhang, J. Thingna, D. He, J.-S. Wang, and B. Li, "Nonlinearity enhanced interfacial thermal conductance and rectification," EPL, vol. 103, no. 6, Article ID 64002, 2013.

[7] S. Ju and X. Liang, "Thermal rectification and phonon scattering in silicon nanofilm with cone cavity," Journal of Applied Physics, vol. 112, no. 5, Article ID 054312, 2012.

[8] N. Yang, S. Hu, D. Ma, T. Lu, and B. Li, "Nanoscale Graphene Disk: A Natural Functionally Graded Material-How is Fourier's Law Violated along Radius Direction of 2D Disk," Scientific Reports, vol. 5, Article ID 14878, 2015.

[9] D. L. Nika, A. I. Cocemasov, D. V. Crismari, and A. A. Balandin, "Thermal conductivity inhibition in phonon engineered coreshell cross-section modulated Si/Ge nanowires," Applied Physics Letters, vol. 102, no. 21, Article ID 213109, 2013.

[10] J. Lee, V. Varshney, A. K. Roy, J. B. Ferguson, and B. L. Farmer, "Thermal rectification in three-dimensional asymmetric nanostructure," Nano Letters, vol. 12, no. 7, pp. 3491-3496, 2012.

[11] A. Özden, A. Kandemir, F. Ay, N. K. Perkgöz, and C. Sevik, "Thermal Conductivity Suppression in Nanostructured Silicon and Germanium Nanowires," Journal of Electronic Materials, vol. 45, no. 3, pp. 1594-1600, 2016.

[12] X. Zhang and $\mathrm{X}$. Wu, "Influence of surface roughness on thermal properties of single crystalline Ge thin films," Computational Materials Science, vol. 123, pp. 40-43, 2016.

[13] A. Malhotra and M. Maldovan, "Impact of Phonon Surface Scattering on Thermal Energy Distribution of Si and SiGe Nanowires," Scientific Reports, vol. 6, Article ID 25818, 2016.

[14] Y. Ni, S. Xiong, S. Volz, and T. Dumitric, "Thermal transport along the dislocation line in silicon carbide," Physical Review Letters, vol. 113, no. 12, Article ID 124301, 2014.

[15] P. Chantrenne and K. Termentzidis, "Prediction of the thermal conductivity of $\mathrm{SiC}$ nanowires with kinetic theory of gases," Physica Status Solidi (A) Applications and Materials Science, vol. 209, no. 12, pp. 2492-2498, 2012.

[16] Q. Li, C.-A. Wang, and S. Tie, "Synthesis of bamboo-like $\mathrm{SiC}$ whiskers from waste silica fume," Crystal Research and Technology, vol. 49, no. 5, pp. 290-297, 2014.

[17] Y. Chu, H. Li, Q. Fu, L. Qi, and L. Li, "Bamboo-shaped SiC nanowire-toughened $\mathrm{SiC}$ coating for oxidation protection of C/C composites," Corrosion Science, vol. 70, pp. 11-16, 2013.

[18] J. Zhu, J. Jia, F. Kwong, and D. H. Ng, "Synthesis of 6H$\mathrm{SiC}$ nanowires on bamboo leaves by carbothermal method," Diamond and Related Materials, vol. 33, pp. 5-11, 2013.

[19] H. Li, X. Yang, Y. Chu, L. Li, Q. Fu, and L. Qi, "Oxidation protection of $\mathrm{C} / \mathrm{C}$ composites with in situ bamboo-shaped $\mathrm{SiC}$ nanowire-toughened Si-Cr coating," Corrosion Science, vol. 74, pp. 419-423, 2013. 
[20] L. Zeng, V. Chiloyan, S. Huberman et al., "Monte Carlo study of non-diffusive relaxation of a transient thermal grating in thin membranes," Applied Physics Letters, vol. 108, no. 6, Article ID 063107, 2016.

[21] R. Wu, K. Zhou, C. Y. Yue, J. Wei, and Y. Pan, "Recent progress in synthesis, properties and potential applications of SiC nanomaterials," Progress in Materials Science, vol. 72, pp. 160, 2015.

[22] G. Shen, Y. Bando, C. Ye, B. Liu, and D. Golberg, "Synthesis, characterization and field-emission properties of bamboo-like $\beta$-SiC nanowires," Nanotechnology, vol. 17, no. 14, article 019, pp. 3468-3472, 2006.

[23] R. Dong, W. Yang, P. Wu et al., "Effect of reinforcement shape on the stress-strain behavior of aluminum reinforced with $\mathrm{SiC}$ nanowire," Materials and Design, vol. 88, pp. 1015-1020, 2015.

[24] K. Termentzidis, T. Barreteau, Y. Ni et al., "Modulated SiC nanowires: Molecular dynamics study of their thermal properties," Physical Review B - Condensed Matter and Materials Physics, vol. 87, no. 12, Article ID 125410, 2013.

[25] X. Zianni, "Monte Carlo simulations on the thermoelectric transport properties of width-modulated nanowires," Journal of Electronic Materials, vol. 45, no. 3, pp. 1779-1785, 2016.

[26] X. Zianni and P. Chantrenne, "Thermal conductivity of diameter-modulated silicon nanowires within a frequencydependent model for phonon boundary scattering," Journal of Electronic Materials, vol. 42, no. 7, pp. 1509-1513, 2013.

[27] X. Zianni, "Disorder-induced enhancement of the thermoelectric efficiency in diameter-modulated nanowires," Microelectronic Engineering, vol. 112, pp. 235-240, 2013.

[28] E. Blandre, L. Chaput, S. Merabia, D. Lacroix, and K. Termentzidis, "Modeling the reduction of thermal conductivity in core/shell and diameter-modulated silicon nanowires," Physical Review B - Condensed Matter and Materials Physics, vol. 91, no. 11, Article ID 115404, 2015.

[29] R. B. Peterson, "Direct simulation of phonon-mediated heat transfer in a Debye crystal," Transactions - ASME: Journal of Heat Transfer, vol. 116, no. 4, pp. 815-822, 1994.

[30] S. Mazumder and A. Majumdar, "Monte Carlo study of phonon transport in solid thin films including dispersion and polarization," Journal of Heat Transfer, vol. 123, no. 4, pp. 749-759, 2001.

[31] Y. Chen, D. Li, J. R. Lukes, and A. Majumdar, "Monte Carlo simulation of silicon nanowire thermal conductivity," Journal of Heat Transfer, vol. 127, no. 10, pp. 1129-1137, 2005.

[32] D. Lacroix, K. Joulain, and D. Lemonnier, "Monte Carlo transient phonon transport in silicon and germanium at nanoscales," Physical Review B - Condensed Matter and Materials Physics, vol. 72, no. 6, 2005.

[33] A. Mittal and S. Mazumder, "Monte carlo study of phonon heat conduction in silicon thin films including contributions of optical phonons," Journal of Heat Transfer, vol. 132, no. 5, pp. 1-11, 2010.

[34] C. Bera, "Monte Carlo simulation of thermal conductivity of Si nanowire: An investigation on the phonon confinement effect on the thermal transport," Journal of Applied Physics, vol. 112, no. 7, Article ID 074323, 2012.

[35] E. B. Ramayya, L. N. Maurer, A. H. Davoody, and I. Knezevic, "Thermoelectric properties of ultrathin silicon nanowires," Physical Review B - Condensed Matter and Materials Physics, vol. 86, no. 11, Article ID 115328, 2012.

[36] S. Wolf, N. Neophytou, Z. Stanojevic, and H. Kosina, "Monte Carlo Simulations of Thermal Conductivity in Nanoporous Si
Membranes," Journal of Electronic Materials, vol. 43, no. 10, pp. 3870-3875, 2014.

[37] S. Abbar, J. Carlson, H. Duan, and S. Reddy, "Quantum Monte Carlo calculations of the thermal conductivity of neutron star crusts," Physical Review C - Nuclear Physics, vol. 92, no. 4, Article ID 045809, 2015.

[38] K. Kukita and Y. Kamakura, "Monte Carlo simulation of phonon transport in silicon including a realistic dispersion relation," Journal of Applied Physics, vol. 114, no. 15, Article ID 154312, 2013.

[39] R. Wu, R. Hu, and X. Luo, "First-principle-based full-dispersion Monte Carlo simulation of the anisotropic phonon transport in the wurtzite GaN thin film," Journal of Applied Physics, vol. 119, no. 14, Article ID 145706, 2016.

[40] A. Ramiere, S. Volz, and J. Amrit, "Geometrical tuning of thermal phonon spectrum in nanoribbons," Journal of Physics D: Applied Physics, vol. 49, no. 11, Article ID 115306, 2016.

[41] Z. Li, Y. Qian, J. Zhao, T. Yao, D. Tan, and M. Chen, "Synthesis of hexagonal prism-shaped $\mathrm{SiC}$ nanowires from a mixture of bamboo flour and silica xerogel," Optoelectronics and Advanced Materials, Rapid Communications, vol. 9, no. 5-6, pp. 734-737, 2015.

[42] R. Dong, W. Yang, P. Wu et al., "Microstructure characterization of $\mathrm{SiC}$ nanowires as reinforcements in composites," Materials Characterization, vol. 103, article 7838, pp. 37-41, 2015.

[43] V. Jean, S. Fumeron, K. Termentzidis, X. Zianni, and D. Lacroix, "Monte Carlo simulations of phonon transport in Si nanowires with constrictions," International Journal of Heat and Mass Transfer, vol. 86, pp. 648-655, 2015.

[44] D.-S. Tang, Y.-C. Hua, and B.-Y. Cao, “Thermal wave propagation through nanofilms in ballistic-diffusive regime by Monte Carlo simulations," International Journal of Thermal Sciences, vol. 109, pp. 81-89, 2016.

[45] K. Kukita, I. N. Adisusilo, and Y. Kamakura, "Monte Carlo simulation of diffusive-to-ballistic transition in phonon transport," Journal of Computational Electronics, vol. 13, no. 1, pp. 264-270, 2014.

[46] W. A. Page, D. Brandt, and M. E. Msall, "Monte Carlo modeling of phonons at crystal interfaces," Journal of Low Temperature Physics, vol. 176, no. 3-4, pp. 176-181, 2014.

[47] L. N. Maurer, Z. Aksamija, E. B. Ramayya, A. H. Davoody, and I. Knezevic, "Universal features of phonon transport in nanowires with correlated surface roughness," Applied Physics Letters, vol. 106, no. 13, Article ID 133108, 2015.

[48] Z. Wang, Z. Ni, R. Zhao, M. Chen, K. Bi, and Y. Chen, "The effect of surface roughness on lattice thermal conductivity of silicon nanowires," Physica B: Condensed Matter, vol. 406, no. 13, pp. 2515-2520, 2011.

[49] V. N.-S. Bong and B. T. Wong, "The effect of phonon anisotropic scattering on the thermal conductivity of silicon thin films at $300 \mathrm{~K}$ and 400K," Journal of Physics and Chemistry of Solids, vol. 88, pp. 41-46, 2016.

[50] J. Rickman, M. Harmer, and H. Chan, "Grain-boundary layering transitions and phonon engineering," Surface Science, vol. 651, pp. 1-4, 2016.

[51] J. Ziman M, Electrons and Phonons, New York, NY, USA, 1960, Oxford University Press.

[52] C. Dames and G. Chen, "Theoretical phonon thermal conductivity of Si/Ge superlattice nanowires," Journal of Applied Physics, vol. 95, no. 2, pp. 682-693, 2004. 
[53] D. Terris, K. Joulain, D. Lemonnier, D. Lacroix, and P. Chantrenne, "Prediction of the thermal conductivity anisotropy of Si nanofilms: results of several numerical methods," International Journal of Thermal Sciences, vol. 48, no. 8, pp. 1467-1476, 2009.

[54] T. T. T. Nghiêm, J. Saint-Martin, and P. Dollfus, "Electrothermal simulation based on coupled Boltzmann transport equations for electrons and phonons," Journal of Computational Electronics, vol. 15, no. 1, pp. 3-15, 2016.

[55] A. Bauer, J. Kräußlich, L. Dressler et al., "High-precision determination of atomic positions in crystals: The case of $6 \mathrm{H}-$ and 4H-SiC", Physical Review B, vol. 57, no. 5, pp. 2647-2650, 1998.

[56] G. A. Slack, "The Thermal Conductivity of Nonmetallic Crystals," Solid State Physics - Advances in Research and Applications, vol. 34, no. C, pp. 1-71, 1979.

[57] J. Liu and Y. K. Vohra, "Raman modes of $6 \mathrm{H}$ polytype of silicon carbide to ultrahigh pressures: A comparison with silicon and diamond," Physical Review Letters, vol. 72, no. 26, pp. 4105-4108, 1994.

[58] Y.-J. Han and P. G. Klemens, "Anharmonic thermal resistivity of dielectric crystals at low temperatures," Physical Review B, vol. 48, no. 9, pp. 6033-6042, 1993.

[59] Y. Goldberg, E. M. Levinshtein, and L. S. Rumyantsev, Properties of Advanced Semiconductor Materials GaN, AlN, SiC, BN, SiC, SiGe, John Wiley \&amp; Sons, Inc., New York, NY, USA, 2001.

[60] D. Morelli, J. Hermans, C. Beetz, W. Woo S, G. Harris L, and C. Taylor, "Silicon Carbide and Related Materials Institute of Physics Conference Series," in Proceedings of the Silicon Carbide and Related Materials Institute of Physics Conference Series, 1993.

[61] S. Ju and X. Liang, "Thermal rectification and phonon scattering in asymmetric silicon nanoribbons," Journal of Applied Physics, vol. 112, no. 2, Article ID 024307, 2012.

[62] J. Cheh and H. Zhao, "Thermal rectification in asymmetric Ushaped graphene flakes," Journal of Statistical Mechanics: Theory and Experiment, vol. 2012, no. 6, Article ID P06011, 2012. 

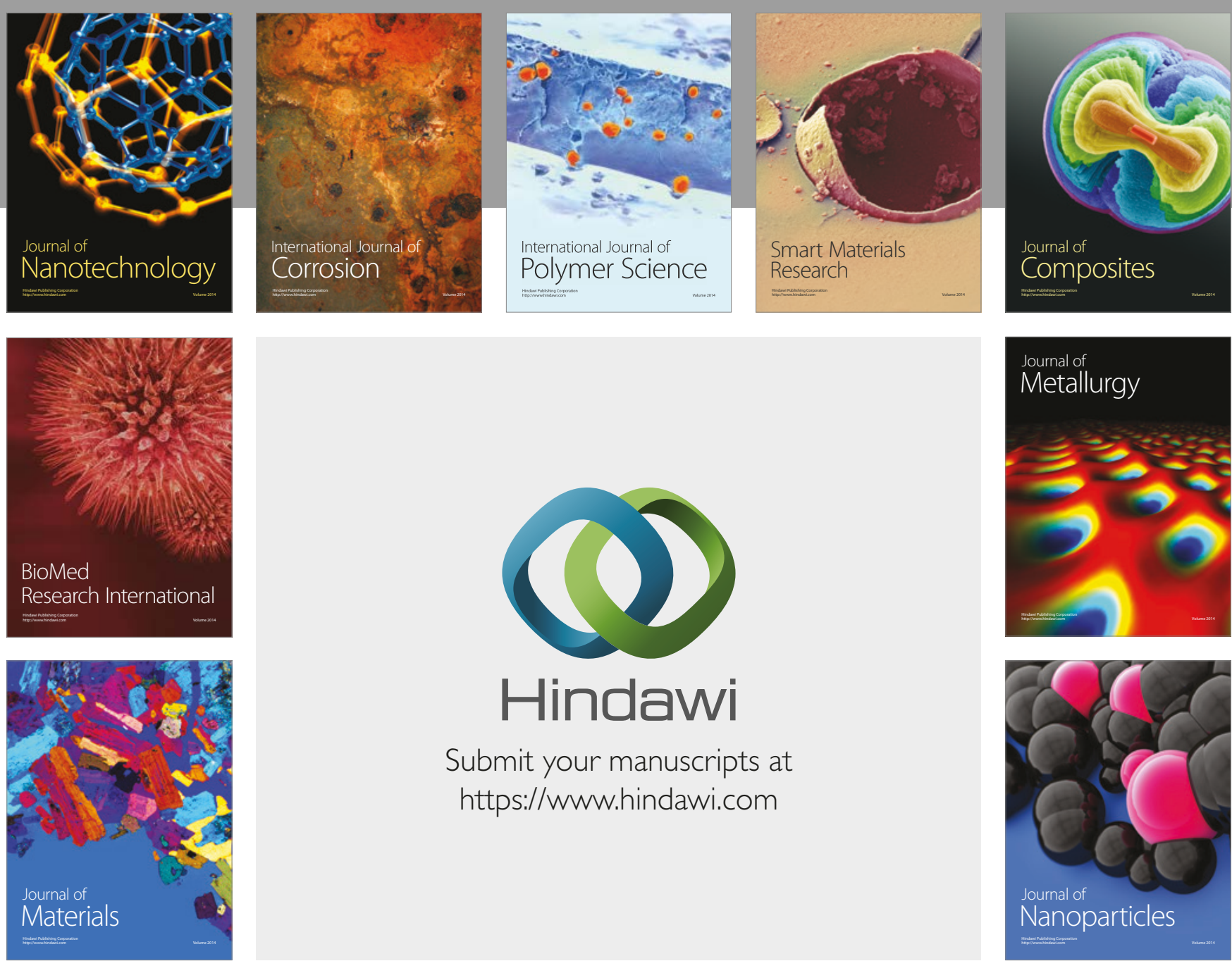

\section{Hindawi}

Submit your manuscripts at

https://www.hindawi.com
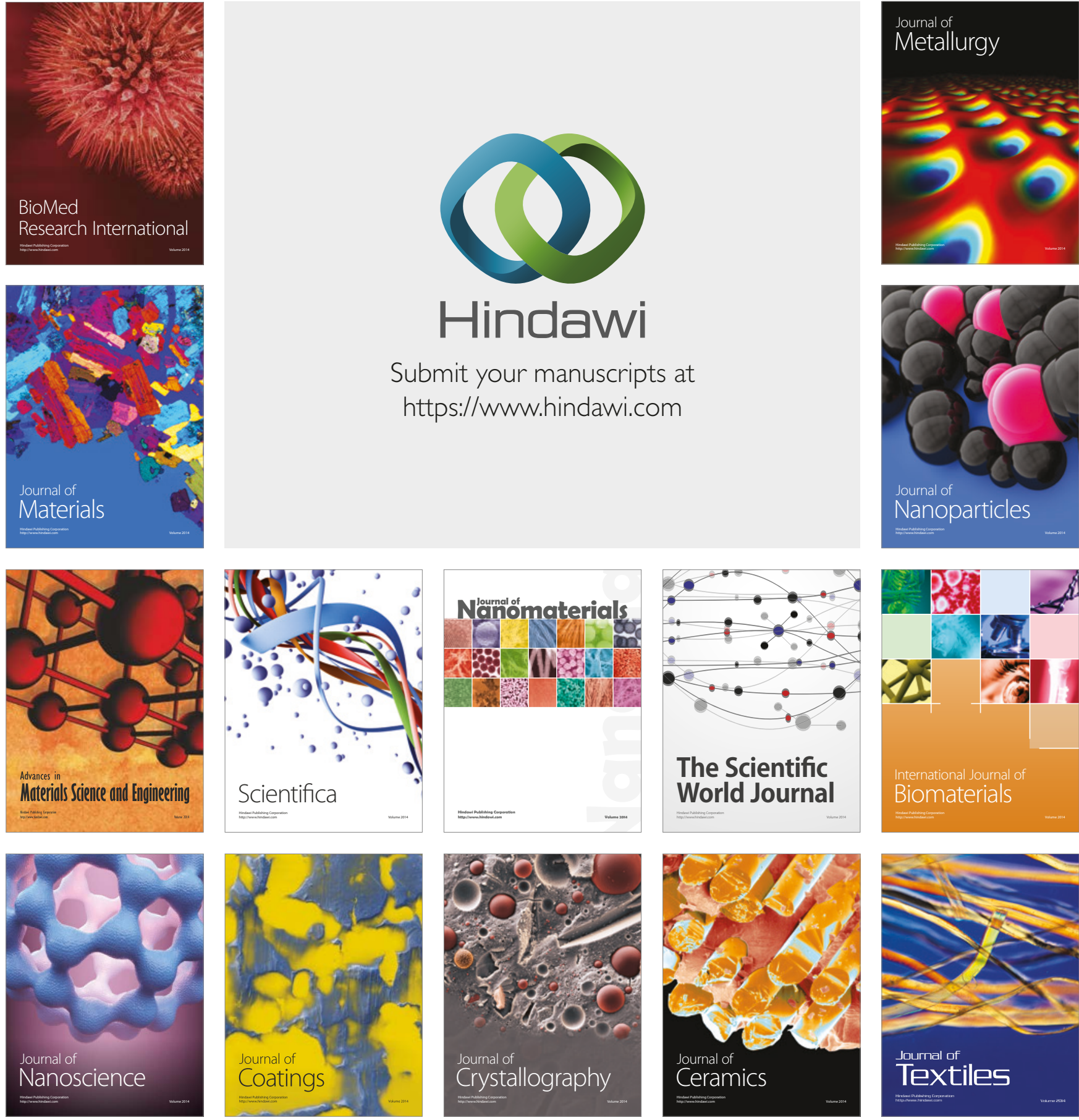

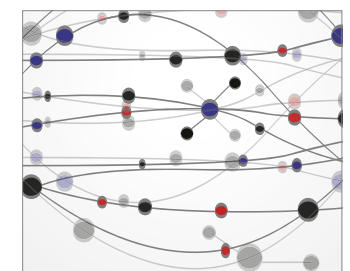

The Scientific World Journal
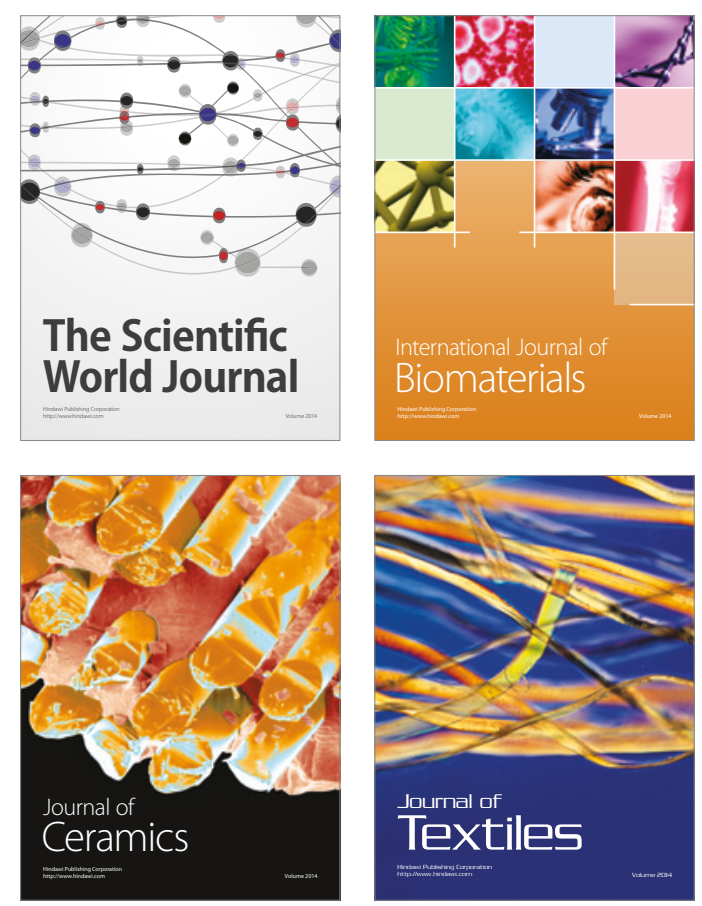